\title{
Failure of cost-benefit analysis in gastrointestinal endoscopy
}

\section{다 (i)}

\author{
Authors \\ Amnon Sonnenberg ${ }^{1,2}$, Gennadiy Bakis ${ }^{3}$ \\ Institutions \\ 1 Gastroenterology Section, Portland VA Medical Center, \\ Portland, Oregon, United States \\ 2 Division of Gastroenterology and Hepatology, Oregon \\ Health \& Science University, Portland, Oregon, United \\ States \\ 3 Pancreaticobiliary Center, Maine Medical Center, \\ Portland, Maine, United States
}

submitted 27.3.2019

accepted after revision 21.6.2019

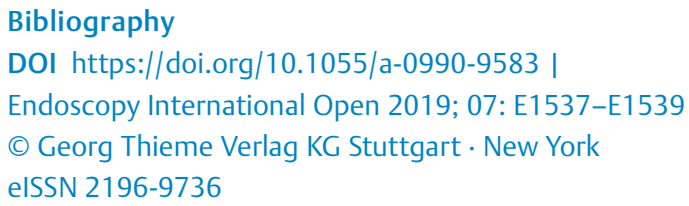

Corresponding author

Amnon Sonnenberg, MD, MSc, Portland VA Medical Center P3-GI, 3710 SW US Veterans Hospital Road, Portland, OR 97239, USA
Fax: +1-503-220-3426

sonnenbe@ohsu.edu

\section{ABSTRACT}

Background and study aims We discuss the occurrence of two cases, where the endoscopic pursuit of diagnostic certainty resulted in adverse events that exceeded the clinical relevance of the endoscopic diagnosis itself. In both instances, physicians were hesitant to subject their patients to a necessary surgical intervention before gastrointestinal endoscopy had provided them with absolute assurance that no other mitigating factors could possibly jeopardize the success of a planned intervention. In trying to avoid a single and potentially bad outcome of a necessary medical intervention, the physicians exposed their patients to many more additional and unnecessary risks. As key players in clinical decision-making, physicians sometimes may find it difficult to disentangle their own risk-benefit considerations from those of their patients.

\section{Introduction}

Compared with other medical interventions, most endoscopic procedures are generally considered minimally invasive. Such assessment is associated with widespread overuse of esophagogastroduodenoscopies (EGD), colonoscopies and other endoscopic procedures for questionable indications [1,2]. Occasionally, patients are subjected to large and potentially lifethreatening risks for a small and hypothetical diagnostic benefit. The pursuit of diagnostic certainty results in adverse events (AEs) that by far exceed the clinical relevance of the diagnosis itself. The two following case scenarios serve to illustrate this point.

\section{Case reports}

The gastroenterology service was consulted regarding a 72year-old man with acute cholecystitis and a cystic duct stone as evidenced by computed tomography (CT) scan and abdomi- nal ultrasound. Both imaging studies also revealed a common bile duct of normal caliber and a largely distended gallbladder with its wall thickened. He had leukocytosis $\left(16000 / \mathrm{mm}^{3}\right)$ and his total bilirubin was $1.5 \mathrm{mg} / \mathrm{dL}$, alkaline phosphatase $147 \mathrm{IU} / \mathrm{L}$, aspartate aminotransferase $55 \mathrm{lU} / \mathrm{L}$, and alanine aminotransferase $38 \mathrm{IU} / \mathrm{L}$.

The gastroenterologists recommended cholecystectomy, but the surgeons were hesitant to operate on him before presence of choledocholithiasis had been completely ruled out [3, 4]. Because of a cardiac pacemaker for atrioventricular block with atrial fibrillation, he could not undergo magnetic resonance cholangiography. The interventional endoscopist felt that the indication for an endoscopic ultrasound (EUS) plus endoscopic retrograde cholangiopancreatography (ERCP) was relatively weak and such endoscopic procedures would have a low yield. Because the patient was on Plavix, the interventional endoscopist recommended withholding this medication for 7 days before reevaluating the need for an EUS plus ERCP. However, in the meantime, the patient's clinical picture worsened, 
with development of fever and more severe abdominal pain. Eventually, interventional radiology placed a percutaneous cholecystostomy, which resulted in multiple large subcapsular hematomas of the liver with hemorrhagic and septic shock. The patient's anticoagulant had to be stopped altogether, and he subsequently also suffered a severe stroke with hemiparesis and decreased mental status.

The second scenario concerned a 48-year-old male with esophageal perforation and atrio-esophageal fistula following radiofrequency ablation for treatment of atrial fibrillation. After cardiac ablation the patient developed chest pain and hematemesis and had a transient ischemic attack (TIA). Thoracic CT scan revealed an esophageal fistula and mediastinal free air. At that point, the surgeons were already quite sure about the patient's primary diagnosis and its relationship to his TIA. Nevertheless, they requested an EGD (with carbon dioxide insufflation) to rule out other gastrointestinal bleeding sites. The endoscopy revealed a stomach filled with blood and a bleeding lesion in the esophagus. After the endoscopy, the patient suffered a stroke with hemiparesis resulting from air embolism [5].

\section{Discussion}

In retrospect, both surgical teams were hesitant to perform the necessary operation before being given absolute assurance that no other mitigating factors would possibly jeopardize the success of their planned operation. The probability of any such mitigating factors was a priori very low. In their attempts to rule out any other hypothetical and minor risk for the surgical procedure, the physicians subjected their patients to more severe AEs than those they were trying to avoid. These cases seem to reflect a general pattern, where the pursuit of diagnostic certainty results in AEs that exceed the clinical relevance of the diagnosis itself. Other such scenarios include requests to rule out upper and lower gastrointestinal bleeding sites in patients with serious comorbid conditions. Frequently, such patients present without any signs or symptoms suggestive of gastrointestinal bleeding, but other preexisting medical conditions that would already suffice to explain the anemia.

For any endoscopic procedure, its expected benefit should exceed its expected cost, that is: $p_{b} \cdot B>p_{c} \cdot C$, where $p_{b}$ and $p_{c}$ represent the probability for occurrence of a beneficial $(B)$ or adverse (and costly) outcome $(C)$, respectively [6]. Here, the term "benefit" is used to indicate all the factors that influence the decision in favor of endoscopy, such as diagnostic and therapeutic gains leading to improvement in patient discomfort and fear. Similarly, the term "cost" includes not only procedural and professional fees, but also risk of potential AEs and their effects on patient well-being [6]. Multiple factors could be responsible for why such comparisons of cost and benefit may fail in clinical practice. At the time of decision-making, physicians may be ignorant about the entire spectrum of potential costs and benefits. Physicians may have difficulty predicting all possible AEs and estimating their probability of occurrence. They may harbor an irrational attachment to absolute certainty, when in clinical practice high probabilities, such as $80 \%$ to $90 \%$, about the occurrence or absence of medical events would already suffice [7].

The greatest hindrance in utilizing cost-benefit analysis may stem from the inability to separate cost-benefit considerations from a physician's and patient's perspective. In both scenarios described here, the physicians were hesitant to embark on operations that were a priori associated with the possibility of a poor outcome. They were focused on optimizing operative preconditions at the expense of all other considerations and thus became more risk averse than the patients themselves might have been. Most decisions on how to advance disease management are largely influenced by the physician's own perspective. As key players in clinical decision-making, physicians may find it difficult or impossible to disentangle their own cost-benefit considerations from those of their patients. This may apply especially to physicians engaged in endoscopic, surgical or other interventional procedures, where procedural expertise and familiarity with their instruments and techniques breeds a bias towards applying their crafts [8]. For a cost-benefit analysis to function, it must not mix the costs and benefits to the physician with those to the patient. For instance, a medical procedure associated with great financial cost to the patient might be beneficial to the physician's income. This poses a general dilemma underlying all decision-making with no easy means at its resolution.

\section{Conclusion}

If cost-benefit considerations are to be useful as a bedside tool in medial decision-making, physicians need to be careful not to fall prey to their own preferences, fears, and gains when advising their patients in difficult decision-making.

\section{Competing interests}

None

References

[1] Hassan C, Di Giulio E, Marmo R et al. Appropriateness of the indication for colonoscopy: systematic review and meta-analysis. J Gastrointest Liver Dis 2011; 20: 279-286

[2] Rubenstein JH, Pohl H, Adams MA et al. Overuse of repeat upper endoscopy in the Veterans Health Administration: a retrospective analysis. Am J Gastroenterol 2017; 112: 1678 - 1685

[3] Rábago LR, Vicente C, Soler F et al. Two-stage treatment with preoperative endoscopic retrograde cholangiopancreatography (ERCP) compared with single-stage treatment with intraoperative ERCP for patients with symptomatic cholelithiasis with possible choledocholithiasis. Endoscopy 2006; 38: 779-786

[4] Schwed AC, Boggs MM, Pham XD et al. Association of admission laboratory values and the timing of endoscopic retrograde cholangiopancreatography with clinical outcomes in acute cholangitis. JAMA Surg 2016; 151: $1039-1045$

[5] Kapur S, Barbhaiya C, Deneke T et al. Esophageal injury and atrioesophageal fistula caused by ablation for atrial fibrillation. Circulation 2017; $136: 1247-1255$ 
[6] Sonnenberg A, Pohl H. 'Do no harm': an intuitive decision tool to assess the need for gastrointestinal endoscopy. Endosc Int Open 2019; 7: E384-E388

[7] Kahneman D, Tversky A. Prospect theory: an analysis of decision under risk. Econometrica 1979; 47: 263-292
[8] Wikipedia contributors. Law of the instrument. Wikipedia, The Free Encyclopedia. Available at https://en.wikipedia.org/wiki/Law_of_the_instrument [Accessed Jan 27, 2019] 